\title{
La décompression, alternative conservatrice pour le traitement des grands kystes des maxillaires : présentation d'un cas
}

\author{
Amadio B, Courtois B, Cousty S, L'Homme A, Duran D \\ Département de Médecine buccale et Chirurgie buccale, Faculté d’Odontologie, Toulouse, France \\ sabeeamadio@hotmail.com
}

Les kystes odontogènes développementaux atteignent parfois des dimensions considérables. Leur traitement, toujours chirurgical, peut être conservateur ou radical. La décompression, ou technique de marsupialisation décrite dès 1882 par Partsch, représente une alternative conservatrice pour le traitement des grands kystes des maxillaires.

Le cas rapporté est celui d'un patient présentant un volumineux kératokyste mandibulaire asymptomatique, découvert de façon fortuite lors d'un examen radiologique. La lésion qui est particulièrement étendue, entretient des rapports étroits avec le nerf alvéolaire inférieur et les apex de toutes les dents entre 36 et 43 . Un traitement par décompression est retenu. La ponction du liquide kystique et la biopsie de la paroi confirment le diagnostic de kératokyste odontogène parakératosique.

Le caractère bénin de la lésion autorise la pose d'un drain mettant en communication la cavité kystique avec le milieu buccal. Il est suturé à la muqueuse et laissé en place le temps nécessaire pour obtenir une involution suffisante. Il va également servir de mainteneur d'espace, empêchant la fermeture de l'orifice et constituer une voie d'accès pour l'irrigation antiseptique quotidienne effectuée par le patient. Après 13 mois de décompression, le second temps chirurgical est effectué : la paroi kystique résiduelle, nettement épaissie et plus résistante, est aisément énucléée. L'examen anatomopathologique confirme l'absence de processus malin. Cinq ans après l'intervention, il n'y a aucun signe de récidive.

La décompression constitue une solution intéressante à la morbidité chirurgicale inhérente à l'exérèse radicale des volumineuses lésions des maxillaires (perte de substance de grande étendue, troubles fonctionnels et préjudice esthétique) séquelles qui semblent inacceptables au regard du caractère bénin de la lésion et du faible taux de récidives obtenus après décompression (Chi 2005, Hupp 2006, Vargas 2007, Tolstunov 2008). Elle répond à des critères chronologiques et techniques stricts et bien codifiés. (Ninomiya, 2002 Kubota, 2004, Oka 2005). De plus, en cas de récidive, il est toujours possible de réintervenir, soit une nouvelle fois de façon conservatrice, soit en adoptant une technique plus radicale (Freidel 1980). 